\title{
Sugar-Induced Release of Guests from Silica Nanocontainer with Cyclodextrin Gatekeepers
}

\author{
Jinwoo Lee, Jeonghun Lee, Saehee Kim, Chang-ju Kim, Sangyong Lee, \\ Byungcheol Min, Yongtae Shin, and Chulhee Kim*
}

\author{
Department of Polymer Science and Engineering, Inha University, Incheon 402-751, Korea. *E-mail: chk@inha.ac.kr \\ Received December 22, 2010, Accepted January 27, 2011
}

Key Words : Cyclodextrin, Mesoporous silica, Sugar, Stimuli-responsive, Controlled release

Stimuli-responsive silica nanoparticles with porous channels have great potential for useful application in the area of drug and gene delivery because of their unique responsiveness and high stability. ${ }^{1-3}$ In particular, these nanoparticles with gatekeepers are numerously attractive as delivery vehicles which have unique properties for the controlled release of guests under specific conditions. ${ }^{4}$ Mesoporous silica nanoparticles (Si-MPs) can serve as outstanding host for guest molecules with various sizes, shapes, and functionalites. ${ }^{5}$ They are more stable under surrounding conditions like $\mathrm{pH}$, temperature than other nanocarriers such as liposomes, polymer micelles. ${ }^{6}$

A variety of gatekeepers have been used to keep guest molecules in the pore before releasing the guest molecules. Recent reports regarding stimuli-responsive nanocontainers have shown that rotaxane, ${ }^{7}$ cyclodextrin $(\mathrm{CD}),{ }^{1,8,9}$ cucurbituril, ${ }^{10}$ polypseudorotaxane, ${ }^{9}$ gold nanoparticle, ${ }^{11}$ antibody, ${ }^{12}$ dendrimer, ${ }^{13,14}$ magnetic nanoparticle ${ }^{15}$ or anion ${ }^{16}$ can be used as the stimuli-responsive gatekeepers on the surface of Si-MPs. There have been various stimuli to remove the gatekeepers such as $\mathrm{pH},{ }^{9,17}$ light $^{8}{ }^{\text {temperature }},{ }^{18}$ photo, ${ }^{1,19}$ competitive binding, ${ }^{20}$ redox potential ${ }^{21}$ and enzyme. ${ }^{1}$

According to recent reports, boronic acid derivatives are used as fluorescent sugar sensors because of their greater stability. $^{22}$ The formation of boronic acid changes due to the concentration of sugar and $\mathrm{pH}$ of the surrounding medium. ${ }^{23,24}$

Herein, we report on stimuli-responsive silica nano-container with CD gatekeepers that contain boronic acid linker, calcein as guest molecules and can exhibit sugar responsive characteristics in the medium of $\mathrm{pH}$ 9.8. The phenylboronic acid derivatives form stable cyclic esters through the strong binding of the boronic acid functionality with the functionality unit of the sugar. ${ }^{25} \mathrm{Si}-\mathrm{PB}$ can form tetrahedral borate intermediates in the $\mathrm{pH}$ level higher than the $\mathrm{p} K_{\mathrm{a}}$ of phenylboronic acid to produce Si-PB-CD in which the CD moiety can be a gatekeeper of the silica nanocontainer. ${ }^{26}$ In addition, removal of the CD moiety from Si-PB-CD by using other sugar such as D-fructose which can bind more strongly to the boronic acid unit than $\mathrm{CD}$ would induce release of the guest molecules in the pore of the silica nanocontainer. The release of the guest molecules can be controlled by $\mathrm{pH}$, nature of the sugar, and the sugar concentration.

For sugar-responsive nanocontainers, we prepared MCM41 type mesoporous silica nanoparticle, Si-MP, with about

$60 \mathrm{~nm}$ diameter (pore diameter $\sim 2 \mathrm{~nm}$ ) and hexagonal porous channels, as shown in Figure 1. As summarized in Scheme 1, we also synthesized aminopropyl-functionalized mesoporous silica nanoparticles $\left(\mathrm{Si}-\mathrm{NH}_{2}\right)$ which contain amine units on the surface. The surface boronic acid unit was introduced by the reaction of the amine group of $\mathrm{Si}-\mathrm{NH}_{2}$ with $p$-dihydroxyborylbenzoic acid $N$-hydroxysuccinimide ester (BA-NHS) to provide Si-PB. The surfactant template (n-cetyltrimethylammonium bromide: CTAB) was removed from Si-PB by refluxing the methanol solution of Si-PB (MeOH/HCl 100:1). The FT-IR spectrum of Si-PB showed the amide absorption band at $1350 \mathrm{~cm}^{-1}$. Si-NH $\mathrm{NH}_{2}$ and $\mathrm{Si}-\mathrm{PB}$ were characterized by transmission electron microscopy (TEM), field emission scanning electron microscope (FESEM), powder X-ray diffraction (XRD) and Barret-JoynerHalenda analysis (BET) (Fig. 1). The $\zeta$-potential values of $\mathrm{Si}-\mathrm{NH}_{2}, \mathrm{Si}-\mathrm{PB}$ and calcein-loaded Si-PB-CD were +14.18 $\mathrm{mV},-12.71 \mathrm{mV}$ and $-22.54 \mathrm{mV}$, respectively.

The quantity of the surface amine groups of $\mathrm{Si}-\mathrm{NH}_{2}$ was estimated to be $0.269 \mathrm{mmol}$ per one gram of $\mathrm{Si}-\mathrm{NH}_{2}$ by ninhydrin test. ${ }^{27}$ After the reaction of $\mathrm{Si}-\mathrm{NH}_{2}$ with BA-NHS, the remaining unreacted amine groups on $\mathrm{Si}-\mathrm{PB}$ were estimated to be $0.0944 \mathrm{mmol}$ per one gram of Si-PB by using the same test. Therefore, the penylboronic acid group
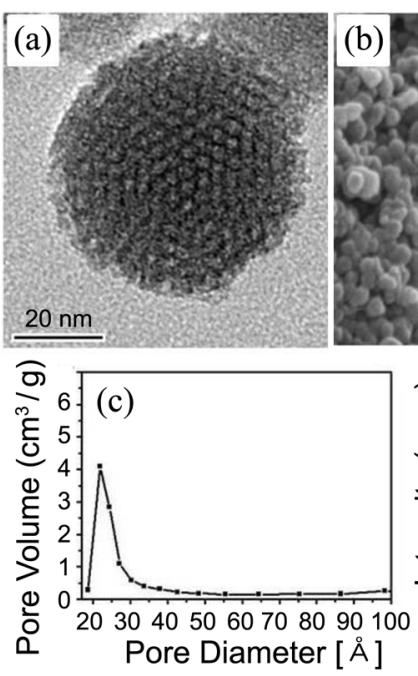

Figure 1. TEM (a) and SEM (b) images of Si-PB. (c) Barret-JoynerHalenda pore distribution of Si-PB, (d) X-ray diffraction pattern of Si-PB. 
(a)
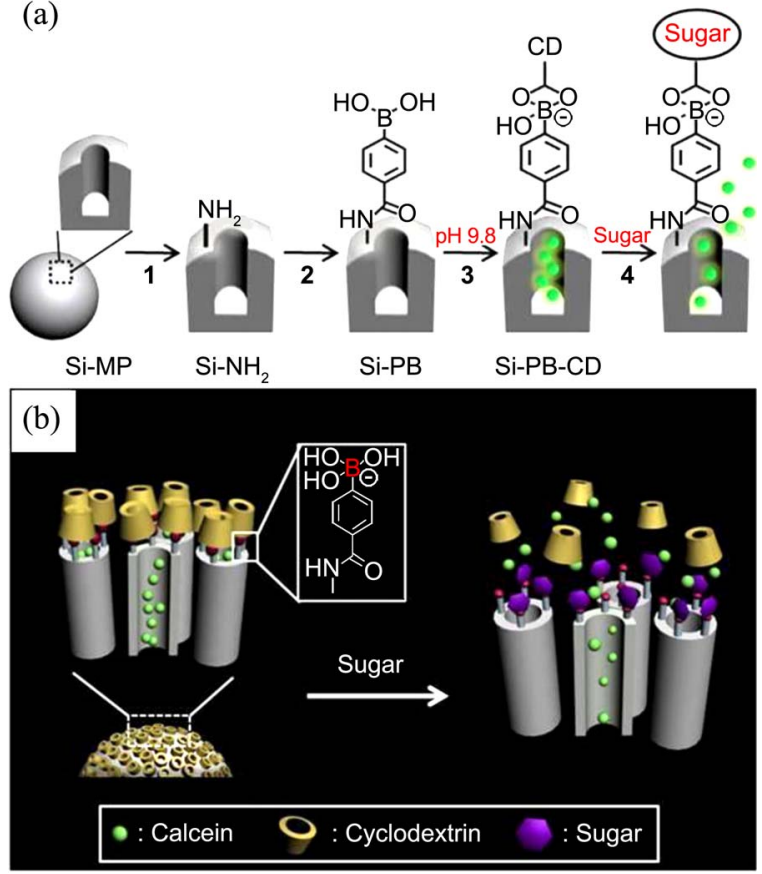

Scheme 1. (a) Synthetic procedure for cyclodextrin-covered nanocontainers (Si-PB-CD). 1) APTES; 2) BANHS; 3) removal of CTAB, loading calcein and capping of the pore with $\beta$-CD in CHES buffer solution ( $\mathrm{pH} 9.8$, adjusted by $\mathrm{NaOH}$ with $0.1 \mathrm{M} \mathrm{NaCl}$ ); 4) addition of sugars such as D-fructose and D-galactose. (b) Schematic illustration of the controlled release of guests from the pore of Si-PB-CD triggered by addition of sugar molecules.

was quantified to be around $0.1746 \mathrm{mmol}$ per one gram of Si-PB. The surface coverage of phenylboronic acid moiety on $\mathrm{Si}-\mathrm{PB}$ was estimated to be about $64.9 \%$. The guest molecules dissolved in the dimethylformamide (DMF) and $10 \mathrm{mM}$ 2-(cyclohexylamino)ethanesulfonic acid (CHES) solution ( $\mathrm{pH} 9.8$, adjusted by $\mathrm{NaOH}$ with $0.1 \mathrm{M} \mathrm{NaCl}$ ) were loaded into the pore of silica nanocontainers by soaking method. ${ }^{28}$ The $\mathrm{pH}$ of the solution was higher than the $\mathrm{p} K_{\mathrm{a}}$ value of the boronic acid unit so that addition of the $\beta-\mathrm{CD}$ would induce the diol coupling with Si-PB. In the condition, a tetragonal boronate ester form of $\beta-C D$ would be an effective gatekeeper of the pore of the nanosilica container.

The weight percentage of $\beta-C D$ on Si-PB-CD measured by a glucose assay kit (Sigma) was $0.56 \%$. The surface coverage of $\beta$-CD on Si-PB-CD, compared with a closely packed surface system, was estimated to be about $10 \% .{ }^{1}$ The loading percentage of calcein in Si-PB-CD calculated by $\mathrm{UV} /$ vis absorption spectra was about $1.8 \mathrm{wt} \%$. The release of the calcein guest molecules from the pore of Si-PB-CD was monitored by fluorescence measurements.

The release property of the guest molecules from the pore of Si-PB-CD was observed by using fluorescence measurements. As shown in Figure 2(a), the calcein guest molecules were not released from the pore of Si-PB-CD more than 6 hours in the absence of D-fructose, indicating that the $\mathrm{CD}$ gatekeeper on Si-PB-CD is very effective to keep the calcein molecules in the pore of Si-PB-CD. As shown in Figure 2(b), the change of the $\mathrm{pH}$ value of the solution lower than
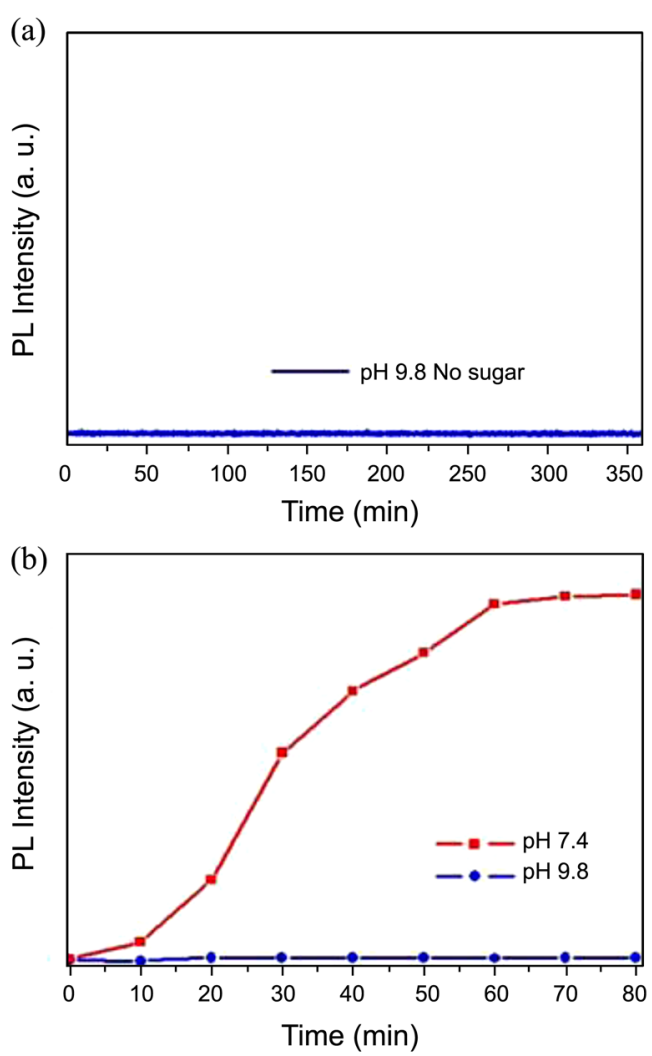

Figure 2. (a) Time-courses of fluorescence intensity of Si-PB-CD in the absence of sugar at $\mathrm{pH}$ 9.8. (b) $\mathrm{pH}$-induced release of guest entrapped in Si-PB-CD at pH 7.4 (red dot) and pH 9.8 (blue dot).

$\mathrm{p} K_{\mathrm{a}}$ of the phenylboronic acid unit induced the release of the guest calcein from Si-PB-CD because the CD unit would be dissociated from the borate ester moiety to open the pore of the silica nanocontainer. ${ }^{26}$

Addition of various sugar molecules such as D-fructose and D-galactose to the aqueous suspension of the calcein loaded Si-PB-CD efficiently triggered release of the guest molecules from the pore, because the $\mathrm{CD}$ gatekeeper on SiPB-CD would be removed by the incoming sugar as shown in Scheme 1(a) and Figure 2(a). D-Fructose and D-galactose bind to the boronic acid moiety more strongly than $\mathrm{CD}$ as shown in Figure 3(a). Because CDs are cyclic oligosaccharides composed of $\alpha-1,4$-coupled D-glucose units, ${ }^{29} \mathrm{D}$ mannose and D-glucose cannot remove the CD gatekeeper as shown in Figure 3(a). The fluorescence intensity of calcein from Si-PB-CD was effectively increased in the high concentration of D-fructose, as shown in Figure 3(b). With increasing the concentration of D-fructose, we can observe more enhanced release of the calcein molecules from the silica nanocontainer.

We also synthesized and investigated the silica nanocontainers capped with $\alpha-$ or $\gamma$-CD. As shown in Figure 4, in the absence of D-fructose, the fluorescence intensities were nearly constant over 2 hours, which indicates that the $\alpha$ - and $\gamma$-CD gatekeeper forcibly blocked release of loaded calcein molecules in the channel of Si-PB-CD in basic condition (pH 9.8). In the presence of $70 \mathrm{mM}$ D-fructose, the entrapped guest molecules in the Si-PB-CD using $\alpha$ - or $\gamma$ - 

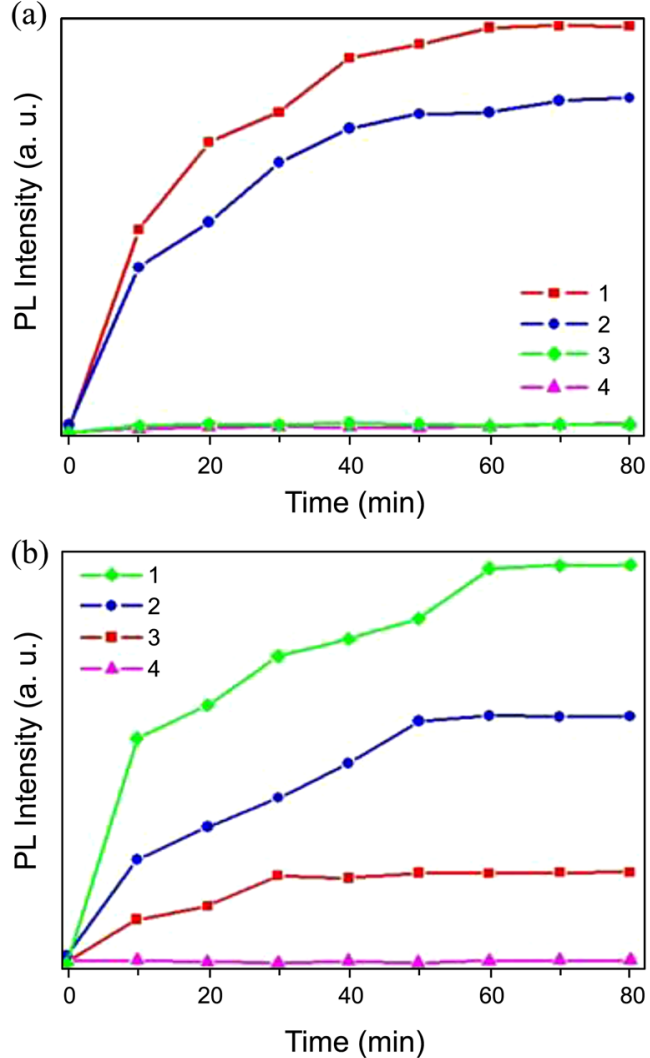

Figure 3. (a) Sugar-induced release of guest from Si-PB-CD; (1) Dfructose, (2) D-galactose, (3) D-mannose, (4) D-glucose. The concentration of monosaccharides was $50 \mathrm{mM}$. (b) Release profiles of guest loaded in Si-PB-CD induced by D-fructose with different concentration; (1) $100 \mathrm{mM}$, (2) $50 \mathrm{mM}$, (3) $10 \mathrm{mM}$, (4) $0 \mathrm{mM}$ of D-fructose.

$\mathrm{CD}$ as gatekeepers were released during about 2 hours as shown in Figure 4. These results indicates that the $\alpha$ - and $\gamma$ $\mathrm{CD}$ are also adequate for gatekeeper of drug carrier to keep guests under control during delivery and replaced by sugar which triggered to release the guest molecules in a controlled manner.

We have demonstrated that the incorporation of the CD gatekeeper on the surface of silica nanocontainers modified with boronic acid is a very efficient approach not only to entrap the guest molecule in the pore reservoir but also to release the guest in response to $\mathrm{pH}$ and the sugar molecules such as D-fructose and D-galactose. In such conditions, the $\mathrm{CD}$ gatekeeper can be well removed at the phenylboronic acid stalk moiety and then the guest molecules were effectively released from Si-PB-CD. We expect that the Si-MPs systems described here can be used as sugar sensitive nanocontainers and provide diverse applications as an effective delivery system.

\section{Experimental}

Materials. Cetyltrimethylammonium bromide (CTAB), tetraethylorthosilicate (TEOS), 3-aminopropyltriethoxysilane (APTES), 4-carboxyphenylboronic acid, $N, N$-diisopropylethylamine (DIPEA), $\mathrm{N}$-hydroxysuccinimide (NHS), $\mathrm{N}$-(3-
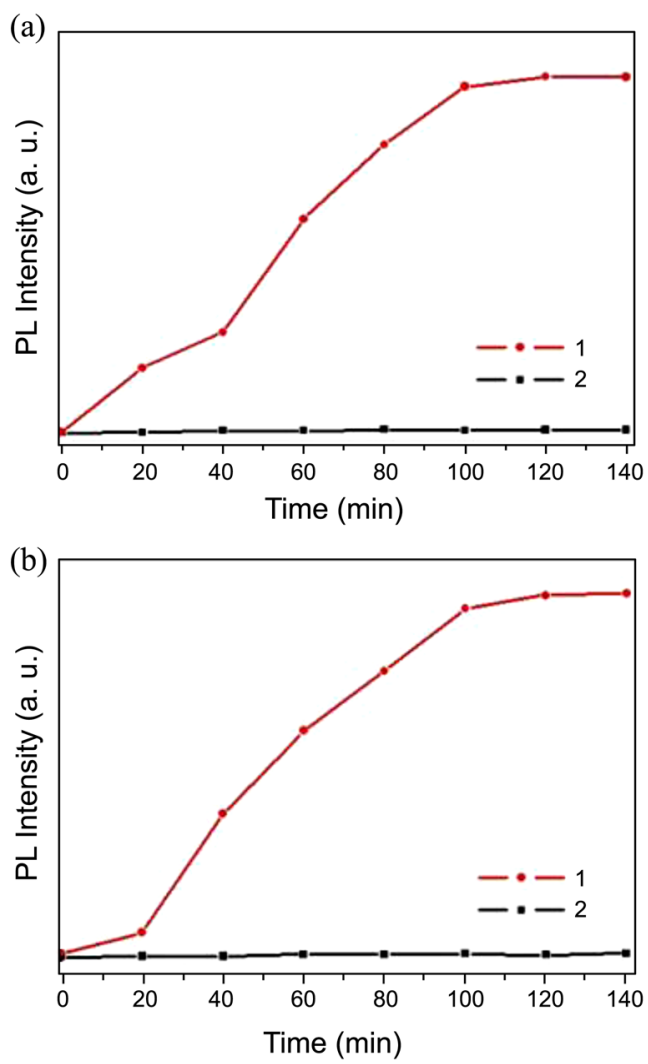

Figure 4. Time courses of fluorescent intensity of Si-PB-CD using $\alpha-C D(a)$ and $\gamma-C D(b)$ as a gatekeeper in the absence (black) and the presence (red) of D-fructose $(70 \mathrm{mM})$ at $\mathrm{pH} 9.8$.

dimethylaminopropyl)- $N$-ethylcarbodiimide hydrochloride (EDC), calcein, ethanol, methanol and dimethylformamide (DMF) were obtained from Aldrich and used as received. D$(+)$-galactose, D-(+)-glucose, D-(-)-fructose, D-(+)-mannose, CHES were obtained from Sigma and used as received. $\beta$ cyclodextrin $(\beta-\mathrm{CD})$ was obtained from TCI and used as received.

Synthesis of Si-MP, Si-NH2. Si-MP and $\mathrm{Si}-\mathrm{NH}_{2}$ were synthesized as described previously. ${ }^{9}$

Synthesis of $\boldsymbol{p}$-Dihydroxyborylbenzoic Acid $N$-Hydroxysuccinimide Ester (BA-NHS). BA-NHS was synthesized using the reference procedure. ${ }^{31}$

Synthesis of Si-PB. Si-NH ${ }_{2}(100 \mathrm{mg})$ in DMF $(20 \mathrm{~mL})$ was stirred for 48 hours at $60{ }^{\circ} \mathrm{C}$ after addition of BA-NHS $(200 \mathrm{mg})$. The resulting particles were separated by centrifugation (9500 rpm, $15 \mathrm{~min}$ ) and washed with DMF (1 time), methanol (2 times) and distilled water (2 times). Then the particles dried in vacuo. To remove CTAB, the resulting particles were stirred for $24 \mathrm{~h}$ at $60{ }^{\circ} \mathrm{C}$ in the mixture of methanol $(16 \mathrm{~mL})$ and concentrated $\mathrm{HCl}(0.9 \mathrm{~mL})$. The surfactant removed nanoparticles were purified by centrifugation and washed several times with methanol.

Synthesis of Si-PB-CD. Si-PB $(30 \mathrm{mg})$ and calcein $(10$ $\mathrm{mg}$ ) suspended in a mixture of CHES buffer $(1 \mathrm{~mL}, \mathrm{pH} 9.8)$ and DMF $(1 \mathrm{~mL})$ were stirred for $24 \mathrm{~h}$ at room temperature. Then, $\beta$-CD $(50 \mathrm{mg})$ was added following to adjust $\mathrm{pH}$ of the solution to 9 with $\mathrm{NaOH}$. The mixture was stirred at 
room temperature for 3 days. The resulting particles were then purified by centrifugation $(9,500 \mathrm{rpm}, 20 \mathrm{~min})$ and washed several times with DMF/CHES solution.

Determination of the Surface Coverage of Amine Groups on Si-MPs. The surface amine groups were quantified at $0.269 \mathrm{mmol}$ per one gram of $\mathrm{Si}-\mathrm{NH}_{2}$ by ninhydrin test. ${ }^{27} \mathrm{In}$ $\mathrm{Si}-\mathrm{PB}$, the remaining surface amine groups were quantified at $0.0944 \mathrm{mmol}$ per one gram of Si-PB by ninhydrin test, and surface boronic acid groups were calculated to be around $0.1746 \mathrm{mmol}$ per one gram of Si-PB by subtracting the amount of remaining surface amine groups from that on Si-PB surface. The surface coverage of phenylboronic acid moiety on Si-PB was estimated to be about $64.9 \%$.

Determination of the Surface Coverage of CDs on SiPB-CD. The quantitative analysis of $\beta-\mathrm{CD}$ on Si-PB-CD was carried out by using glucose assay kit (Sigma) based on the manufacturer's procedure. The weight percentages of $\beta$ $\mathrm{CD}$ on Si-PB-CD were $0.56 \mathrm{wt} \%$. The surface coverage of $\beta-C D$ on Si-PB-CD, compared with a closely packed surface system, was estimated to be about $10 \%{ }^{1}$

Instrument.

Fluorescence Measurements. All the fluorescence measurements were performed using a Shimadzu RF-5301PC spectrofluorophotometer. The emission and excitation slit widths were set at $3 \mathrm{~nm}$, and the excitation wavelength was $490 \mathrm{~nm}$ (the absorption maximum of calcein).

X-ray Diffraction (XRD) Experiment. XRD patterns were recorded at room temperature on a Rigaku model MAX $2200 \mathrm{~V}$ counter diffractometer with a $\mathrm{CuK}_{\alpha}$ radiation source (Operated at $60 \mathrm{kV}, 60 \mathrm{~mA}$ ).

Barret-Joyner-Halenda Pore Distribution (BET). The pore size was measured at $77 \mathrm{~K}$ on a Quantachrome instrument.

Transmission Electron Microscopy (TEM) Analysis. TEM images were obtained using a Philips CM 200 instrument operated at an acceleration voltage of $120 \mathrm{kV}$. For the preparation of dispersed samples in water, a drop of sample solution $(100 \mathrm{mg} / \mathrm{L})$ was placed onto a $300-$ mesh copper grid coated with carbon. About 2 min after deposition, the grid was touched with filter paper to remove surface water. The samples were air dried before measurement.

Field Emission Scanning Electron Microscope (FESEM) Analysis. FE-SEM analysis was carried out on a field emission gun FE-SEM instrument (Hitachi S-4200) with 10$15 \mathrm{kV}$ of accelerating voltage and 0.8-0.9 Torr of pressure range. The FE-SEM samples were prepared by transferring a drop of sample solution onto a 200 mesh carbon-coated copper grid or a silicon wafer. About 5-10 min after deposition, excess water was removed by touching the edge of the substrate with filter paper. The samples were air dried before measurement.

Zeta-potential Analysis. Zeta-potential values were obtained using OTSUKA Particle Size Analyzer ELS-Z2 by dispersing the sample in distilled water.

Fourier Transform Infrared Spectroscopy (FT-IR). FTIR spectra were obtained using Perkin-Elmer System 2000 FR-IR spectrophotometer.

UV/vis Spectra. UV/vis spectra were recorded on HewlettPackard 8452A spectrophotometer.
Acknowledgments. This work was supported by the Korea Research Foundation (KRF) grant funded by the Korea government (MEST) (No. 2009-0079739).

\section{References}

1. Park, C.; Kim, H.; Kim, S.; Kim, C. J. Am. Chem. Soc. 2009, 131, 16614.

2. Nguyen, T. D.; Leung, K. C.; Liong, M.; Liu, Y.; Stoddart, J. F.; Zink, J. I. Adv. Funct. Mater. 2007, 17, 2101.

3. Trewyn, B. G.; Slowing, I. I.; Giri, S.; Chen, H.; Lin, V. S.-Y. Acc. Chem. Res. 2007, 40, 846.

4. Shum, P.; Kim, J.; Thompson, D. H. Adv. Drug Deliv. Rev. 2001, 53, 273.

5. Slowing, I. I.; Trewyn, B. G.; Giri, S.; Lin, V. S.-Y. Adv. Funct. Mater. 2007, 17, 1225.

6. Barbé, C.; Bartlett, J.; Kong, L.; Finnie, K.; Lin, H. Q.; Larkin, M.; Calleja, S.; Bush, A.; Calleja, G. Adv. Mater. 2004, 16, 1959.

7. Nguyen, T. D.; Liu, Y.; Saha, S.; Leung K. C.; Stoddart, J. F.; Zink, J. I. J. Am. Chem. Soc. 2007, 129, 626.

8. Park, C.; Lee, K.; Kim, C. Angew. Chem. Int. Ed. 2009, 48, 1275.

9. Park, C.; Oh, K.; Lee, S. C.; Kim, C. Angew. Chem. Int. Ed. 2007, 46, 1455.

10. Angelos, S.; Khashab, N. M.; Yang, Y.; Trabolsi, A.; Khatib, H. A.; Stoddart, J. F.; Zink, J. I. J. Am. Chem. Soc. 2009, 131, 12912.

11. Aznar, E.; Marcos, M. D.; Martínez-Máñez, R.; Sancenón, F.; Soto, J.; Amorós, P.; Guillem, C. J. Am. Chem. Soc. 2009, 131, 6833.

12. Climent, E.; Bernardos, A.; Martínez-Máñez, R.; Maquieira, A.; Marcos, M. D.; Pastor-Navarro, N.; Puchades, R.; Sancenón, F.; Soto, J.; Amorós, P. J. Am. Chem. Soc. 2009, 131, 14075.

13. Radu, D. R.; Lai, C.; Jeftinija, K.; Rowe, E. W.; Jeftinija, S.; Lin, V. S.-Y. J. Am. Chem. Soc. 2004, 126, 13216.

14. Diaz, I.; Garcia, B.; Alonso, B.; Casado, C. M.; Moran, M.; Losada, J.; Perez-Pariente, J. Chem. Mater. 2003, 15, 1073.

15. Giri, S.; Trewyn, B. G.; Stellmaker, M. P.; Lin, V. S.-Y. Angew. Chem. Int. Ed. 2005, 44, 5038.

16. Casasus, R.; Climent, E.; Marcos, M. D.; Martinez-Manez, R.; Sancenon, F.; Soto, J.; Amoros, P.; Cano, J.; Ruiz, E. J. Am. Chem. Soc. 2008, 130, 1903.

17. Yang, Q.; Wang, S.; Fan, P.; Wang, L.; Di, Y.; Lin, K.; Xiao, F. Chem. Mater. 2005, 17, 5999.

18. You, Y.; Kalebaila, K. K.; Brock, S. L.; Oqpický, D. Chem. Mater. 2008, 20, 3354.

19. Patel, K.; Angelos, S.; Dichtel, W. R.; Coskun, A.; Yang, Y.-W.; Zink, J. I.; Stoddart, J. F. J. Am. Chem. Soc. 2008, 130, 2382.

20. Leung, K. C.; Nguyen, T. D.; Stoddart, F.; Zink, J. I. Chem. Mater. 2006, 18, 5919.

21. Liu, R.; Zhao, X.; Wu, T.; Feng, P. J. Am. Chem. Soc. 2008, 130, 14418.

22. Tong, A.; Yamauchi, A.; Hayashita, T.; Zhang, Z.; Smith, B. D.; Teramae, N. Anal. Chem. 2001, 73, 1530.

23. Roy, D.; Cambre, J. N.; Sumerlin, B. S. Chem. Commun. 2008, 21, 2477.

24. Kim, K. T.; Cornelissen, J. J. L. M.; Nolte, R. J. M.; van Hest, J. C. M. Adv. Mater. 2009, 21, 2787.

25. Zhao, Y.; Trewyn, B. G.; Slowing, I. I.; Lin, V. S.-Y. J. Am. Chem. Soc. 2009, 131, 8398.

26. Yan, J.; Springsteen, G.; Deeter, S.; Wang, B. Tetrahedron 2004, $60,11205$.

27. Tsai, M.; Tsai, T.; Shieh, D.; Shiu, H.; Lee, C. Anal. Chem. 2001, 73,1530 .

28. Angelos, S.; Yang, Y.-W.; Patel, K.; Stoddart, J. F.; Zink, J. I. Angew. Chem. Int. Ed. 2008, 47, 2222.

29. Van, D. M.; Vermonden, T.; van Nostrum, C. F.; Hennink, W. E. Biomacromolecules 2009, 10, 3157.

30. Armarego, W. L. F.; Perrin, D. D. Purification of Laboratory Chemicals, 4th ed; Butterworth- Heinemann: Oxford, 1996.

31. Masuda, T.; Nagasaki, T.; Tamagaki, S. Supramolecular Chemistry 2000, 11,301 . 